\title{
Expression and Regulation of Catecholaminergic Traits in Primary Sensory Neurons: Relationship to Target Innervation in vivo
}

\author{
David M. Katz and Ira B. Black \\ Division of Developmental Neurology, Cornell University Medical College, New York, New York 10021
}

Catecholaminergic (CA) phenotypic characteristics have recently been detected in adult sensory neurons, demonstrating that $\mathrm{CA}$ expression in the periphery extends beyond the sympathoadrenal axis. Consequently, we may now determine whether common principles underlie CA phenotypic organization in functionally and embryologically diverse populations of peripheral neurons. To begin defining sensory transmitter regulation, the present study examined the relationship of $\mathrm{CA}$ expression to sensory target innervation in cranial nerve ganglion cells of the adult rat.

Retrograde labeling combined with tyrosine hydroxylase (TH) immunocytochemistry indicated that $80-90 \%$ of CA sensory neurons in the glossopharyngeal petrosal ganglion project peripherally in the carotid sinus nerve (CSN). Most of these cells innervate a single target, the carotid body, revealing a striking correlation between $\mathrm{CA}$ expression and the pattern of sensory target innervation. Furthermore, $\mathrm{CSN}$ transection resulted in a transient marked decrease in $\mathrm{TH}$ catalytic activity and immunoreactivity within 1 week. Activities returned to normal by 3 weeks. Thus, axotomy reversibly decreased sensory TH, reproducing effects observed with central $\mathrm{CA}$ neurons (Ross et al., 1975), but differing in certain aspects from observations with sympathetic CA neurons (Cheah and Geffen, 1973; Kessler and Black, 1979). To determine whether disruption of axonal transport itself decreased TH in petrosal neurons, colchicine cuffs were placed around the intact CSN. Colchicine blockade reproduced the effects of axotomy, suggesting that deranged transport, and not axonal damage per se, altered TH. Finally, we studied the role of sensory projections to the CNS by examining petrosal TH after glossopharyngeal nerve rhizotomy. In contrast to sequelae of peripheral axotomy, rhizotomy did not alter TH, suggesting that projections to the periphery predominate in regulation of sensory TH. Our observations suggest that a number of common mechanisms regulate this specific $C A$ character in diverse neuronal populations.

To elucidate the molecular bases of neuronal phenotypic diversity, we have been studying expression and regulation of catecholaminergic (CA) transmitter characteristics in functionally and embryologically distinct populations of neurons (Jonakait et al., 1984; Katz et al., 1983). These studies, documenting CA traits in cranial ganglion primary sensory neurons, dem-

\footnotetext{
Received July 2, 1985; revised Oct. 1, 1985; accepted Oct. 2, 1985.

This work was supported by a grant-in-aid from the American Heart Association with funds contributed in part by the New York Heart Association, the Dysautonomia Foundation, Inc., NIH Grants NS 10259 and HD 12108, and a McKnight Research Project Award to I.B.B.

Correspondence should be addressed to David M. Katz, Division of Developmental Neurology, Cornell University Medical College, 5155 East 71 st Street, New York, NY 10021.

Copyright (C) 1986 Society for Neuroscience $0270-6474 / 86 / 040983-07 \$ 02.00 / 0$
}

onstrated that CA phenotypic expression in the periphery extends beyond the classically defined sympathoadrenal derivatives of the embryonic neural crest (see also Price and Mudge, 1983). Unlike sympathetic neurons, CA sensory neurons, localized to the vagal, nodose and glossopharyngeal, petrosal ganglia are believed to be derived from embryonic epibranchial placodes (LeDouarin et al., 1981). Thus, developmental lineage, function, and patterns of synaptic connectivity alone do not appear to be determinative of CA phenotypic expression.

It is not known, however, whether common mechanisms regulate individual CA traits in sensory and sympathetic CA populations. To approach this issue, the present study begins defining sensory $\mathrm{CA}$ regulation, and compares regulatory mechanisms with sympathetic neurons. By examining regulation of molecular traits in diverse cell types, underlying principles of CA phenotypic organization may be revealed.

CA primary sensory neurons express catalytically active and immunoreactive tyrosine hydroxylase $(\mathrm{TH})$, the rate-limiting enzyme in catecholamine biosynthesis, in addition to other CA traits (Katz et al., 1983). In sympathoadrenal cells, extracellular signals, including transsynaptic depolarizing stimuli, circulating hormones, and target-derived factors, such as NGF, play critical roles in regulation of CA biosynthetic enzymes (Black, 1978). Moreover, multiple intracellular mechanisms, including regulation of mRNA levels (Mallet et al., 1983), proteolytic degradation (Ciaranello et al., 1976), and enzyme phosphorylation (Haycock et al., 1982), mediate extracellular influences on CA phenotypic characteristics. It is not known, however, whether similar processes regulate sensory CA traits.

Initial studies in our laboratory suggested that peripheral target innervation may play a role in regulating sensory $\mathrm{TH}$ (Katz et al., 1983). Peripheral axotomy, for example, decreased TH catalytic activity and immunocytochemical staining in sensory perikarya. Whether this effect is transient, implying altered regulation of enzyme levels, or simply a reflection of cell death, has not been explored. Moreover, specific tissues innervated by CA sensory neurons have not been identified, limiting our ability to define potential target effects on sensory CA expression. The present study was undertaken, therefore, to define sensory C.A targets, and to elucidate the role of peripheral and central projections in regulation of sensory $\mathrm{TH}$. Comparing $\mathrm{TH}$ regulation in sensory and sympathetic neurons may reveal whether common regulatory mechanisms are exhibited by diverse neuronal populations that express similar phenotypic traits.

\section{Materials and Methods}

Animals

Three- to six-week-old female Sprague-Dawley rats (Hilltop Lab Animals, Scottsdale, PA) were housed as previously described (Katz et al., 1983). 


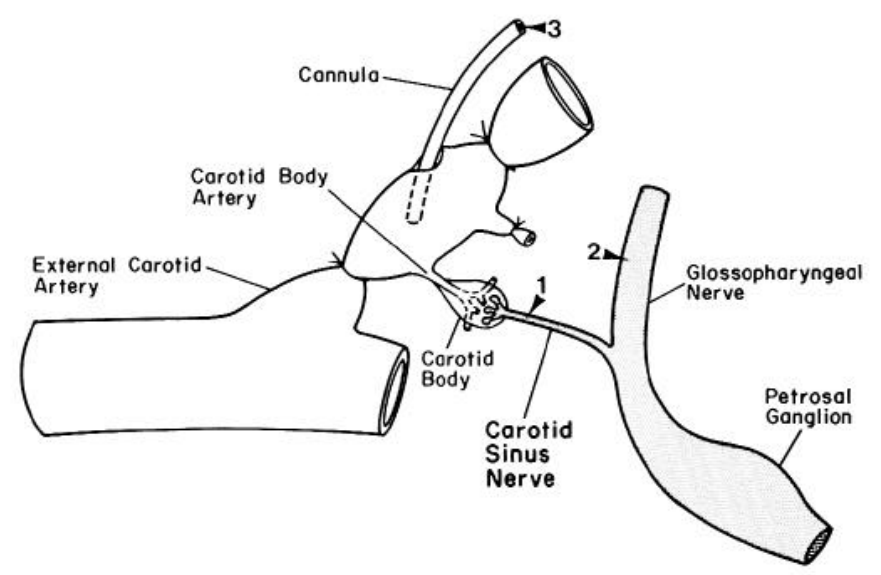

Figure 1. Schematic diagram illustrating sites of bisbenzamide application in retrograde labeling studies. At 1 and 2, tracer was applied to the cut central end of the nerve indicated. At 3, the tracer was infused as described in Materials and Methods.

\section{Surgical procedures}

Animals were anesthetized with halothane and the left carotid bifurcation exposed by a ventral midline incision.

Retrograde transport studies: nerve labeling. The glossopharyngeal nerve, distal to the origin of the carotid sinus nerve (CSN), or the CSN itself, was exposed and transected. The proximal stump was placed on a small piece of Parafilm and covered with a highly concentrated solution of bisbenzamide tetrahydrochloride (Sigma) in distilled water for $1 \mathrm{hr}$. Incisions were sutured and the animals were allowed to recover.

Carotid body infusions. The carotid body was dissected free of all surrounding tissue with the exception of the carotid body artery and CSN. The external carotid artery, above and below the origin of the carotid body artery, and the occipital artery were ligated, creating a partially blind segment of external carotid artery that drained exclusively into the carotid body artery (Fig. 1). This segment was cannulated with polyethylene tubing attached to a syringe. Two percent (wt/vol) bisbenzamide (Sigma) in distilled water was infused via the cannula into the carotid body for approximately $15 \mathrm{~min}$. The cannula was then withdrawn, the artery drained of residual bisbenzamide, and vascular ligatures repositioned to permit resumption of carotid body arterial blood flow.

Carotid sinus nerve transection. For studies of axotomy effects on petrosal ganglion $\mathrm{TH}$, the CSN was exposed and transected as previously described (Katz et al., 1983).

Glossopharyngeal nerve rhizotomy. The extracranial root of the glossopharyngeal nerve was exposed and transected at its exit from the jugular foramen of the skull.

\section{Staining procedures}

Animals were perfused with $200-400 \mathrm{ml}$ of ice-cold $4 \%$ paraformaldehyde in $0.1 \mathrm{~m}$ phosphate buffer, $\mathrm{pH} 7.4$. Tissues were placed in $30 \%$ sucrose $(0.1 \mathrm{~m}$ sodium phosphate buffer, $\mathrm{pH} 7.4)$ overnight at $4^{\circ} \mathrm{C}$ and cut at $15 \mu \mathrm{m}$ on a cryostat, and sections were melted onto gelatin-coated glass slides. Sections were then examined under uv illumination (Leitz Ploem filter cube I) to visualize bisbenzamide fluorescence and photographed. Slides were then heated to $55^{\circ} \mathrm{C}$ for $15 \mathrm{~min}$ and rinsed for $15 \mathrm{~min}$ in sodium PBS ( $0.1 \mathrm{~m}$ sodium phosphate, $0.9 \%$ saline, $\mathrm{pH} 7.4$ ). Sections were processed for $\mathrm{TH}$ immunofluorescence as previously described (Katz et al., 1983).

\section{Morphometric studies}

Drawings of TH-stained sections were traced from projections of 35 $\mathrm{mm}$ transparencies, and each TH-positive cell outlined. The nucleus, if present, was also drawn. The corresponding transparency, showing bisbenzamide labeling in the same section, was then projected onto the initial drawing and the position of all labeled neuronal nuclei marked. The number of TH-positive, bisbenzamide-positive, and double-labeled (TH and bisbenzamide) cells in each ganglion was then determined directly from the composite drawings. To minimize the possibility of counting the same cell more than once in adjacent sections, only cells with a visible nucleus were counted.
Table 1. Summary data, showing the percentage [\% (Bbz, TH/TH)] of tyrosine hydroxylase-containing petrosal sensory neurons retrogradely labeled following bisbenzamide application to the sites indicated

\begin{tabular}{|c|c|c|c|c|c|}
\hline \multirow[b]{2}{*}{ Bisbenzamide labeling site } & \multicolumn{3}{|c|}{$\begin{array}{l}\text { Number of labeled } \\
\text { ganglion cells }(\bar{x})\end{array}$} & \multirow[b]{2}{*}{$\%$} & \\
\hline & $\mathrm{Bbz}$ & TH & $\begin{array}{l}\text { Bbz, } \\
\text { TH }\end{array}$ & & $\frac{\mathrm{Bbz}, \mathrm{TH}}{\mathrm{TH}}$ \\
\hline Carotid sinus nerve $(n=2)$ & 405 & 125 & 116 & 93 & \\
\hline $\begin{array}{l}\text { Distal giossopharyngeal nerve } \\
\qquad(n=2)\end{array}$ & 883 & 124 & 3.5 & 2.9 & \\
\hline Carotid body $(n=2)$ & 395 & 174 & 147 & 84 & \\
\hline
\end{tabular}

Bbz, bisbenzamide-labeled cells; $\mathrm{TH}$, tyrosine hydroxylase-immunoreactive cells; $\mathrm{Bbz}$, Th, double-labeled Bbz- and TH-positive cells. Each value represents the mean of two experiments.

\section{TH catalytic assay}

Tissue samples containing the petrosal ganglion and the rostral onethird of the nodose ganglion (normally fused in situ) were assayed as previously described (Katz et al., 1983).

\section{Colchicine treatment}

Colchicine-impregnated $(0.10 \%)$ Silastic (Dow Corning) nerve cuffs were prepared as previously described in this laboratory (Kessler and Black, 1979).

\section{Statistical analysis}

Data were analyzed by the Student's $t$ test. Multiple data were analyzed by the one-way analysis of variance and the Newman-Keul's test.

\section{Results}

\section{Identification of peripheral targets innervated}

\section{by CA petrosal ganglion cells}

The majority of petrosal ganglion (PG) sensory neurons project peripherally in the glossopharyngeal nerve, and their axons follow one of two primary pathways. The main nerve trunk innervates the upper gastrointestinal tract and tongue, whereas the CSN innervates the carotid body and carotid sinus. We previously found that glossopharyngeal nerve transection proximal, but not distal, to the CSN decreased petrosal TH, suggesting a selective projection of CA afferents in the CSN (Katz et al., 1983). In the present study, retrograde labeling techniques, combined with $\mathrm{TH}$ immunocytochemical staining, were used to identify precisely the peripheral targets innervated by petrosal CA neurons.

Bisbenzamide, a retrogradely transported nuclear dye, was applied to the CSN or to the glossopharyngeal nerve, distal to the origin of the CSN (see Fig. 1). Twenty-four hours after application of bisbenzamide to the CSN, extensive nuclear labeling was observed within neuronal somata in the ipsilateral petrosal ganglion (Fig. 2A). Labeled neurons, numbering approximately 400 per ganglion, were located primarily within the distal one-half to one-third of the PG.

To define the proportion of catecholaminergic petrosal sensory neurons that projects via the CSN, bisbenzamide-labeled sections were photographed and subsequently stained for TH immunoreactivity, using a highly specific antiserum (Markey et al., 1980; see Materials and Methods). As previously reported, numerous immunoreactive sensory perikarya and nerve fibers were observed in the PG. Morphometric analysis demonstrated an average of $125 \mathrm{TH}$-positive cells per ganglion; of these, 116 (93\%) were retrogradely labeled following bisbenzamide application to the carotid sinus nerve (Fig. $2 B$, Table 1).

To determine whether CA sensory neurons project to other 

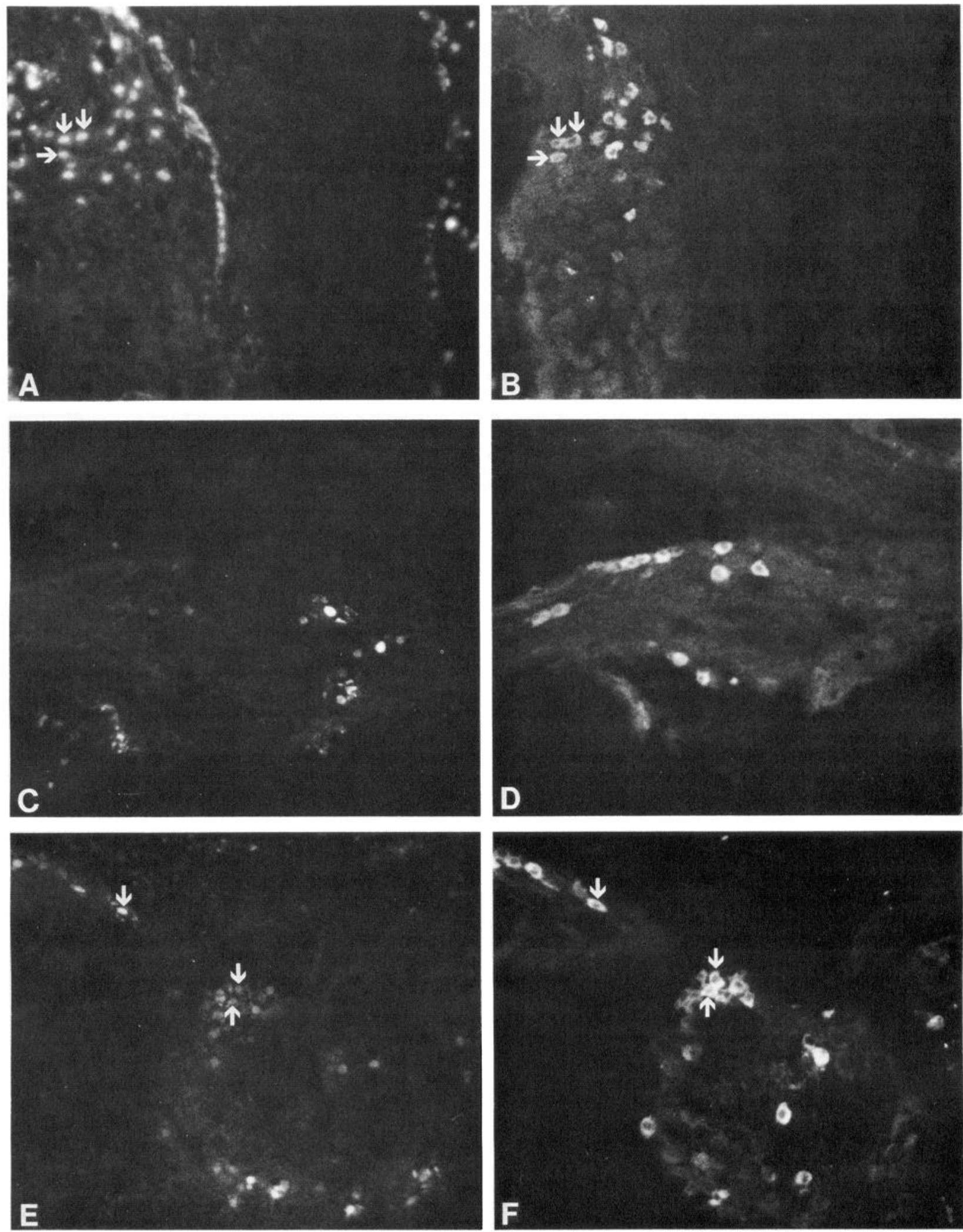

Figure 2. Fluorescence photomicrographs of bisbenzamide labeling $(A, C, E)$ and TH immunoreactivity $(B, D, F)$ within PG neurons 24 hr after bisbenzamide application to the $\operatorname{CSN}(A, B)$, glossopharyngeal nerve, distal to the $\operatorname{CSN}(C, D)$, or infusion into the ipsilateral carotid body $(E$, $F$ ) (see Materials and Methods). The two photomicrographs in each pair show the same tissue section, processed and photographed sequentially as described in Materials and Methods. 
Figure 3. Effect of CSN transection on petrosal TH activity. Enzyme activity is expressed as a percentage of control, using ganglia from sham-operated, agematched animals ( 3 weeks old) for comparison. Each point represents the mean \pm SEM for six to eight determinations. Shaded area, The SE of the grand mean for all control values. ${ }^{*} p<$ 0.01 .

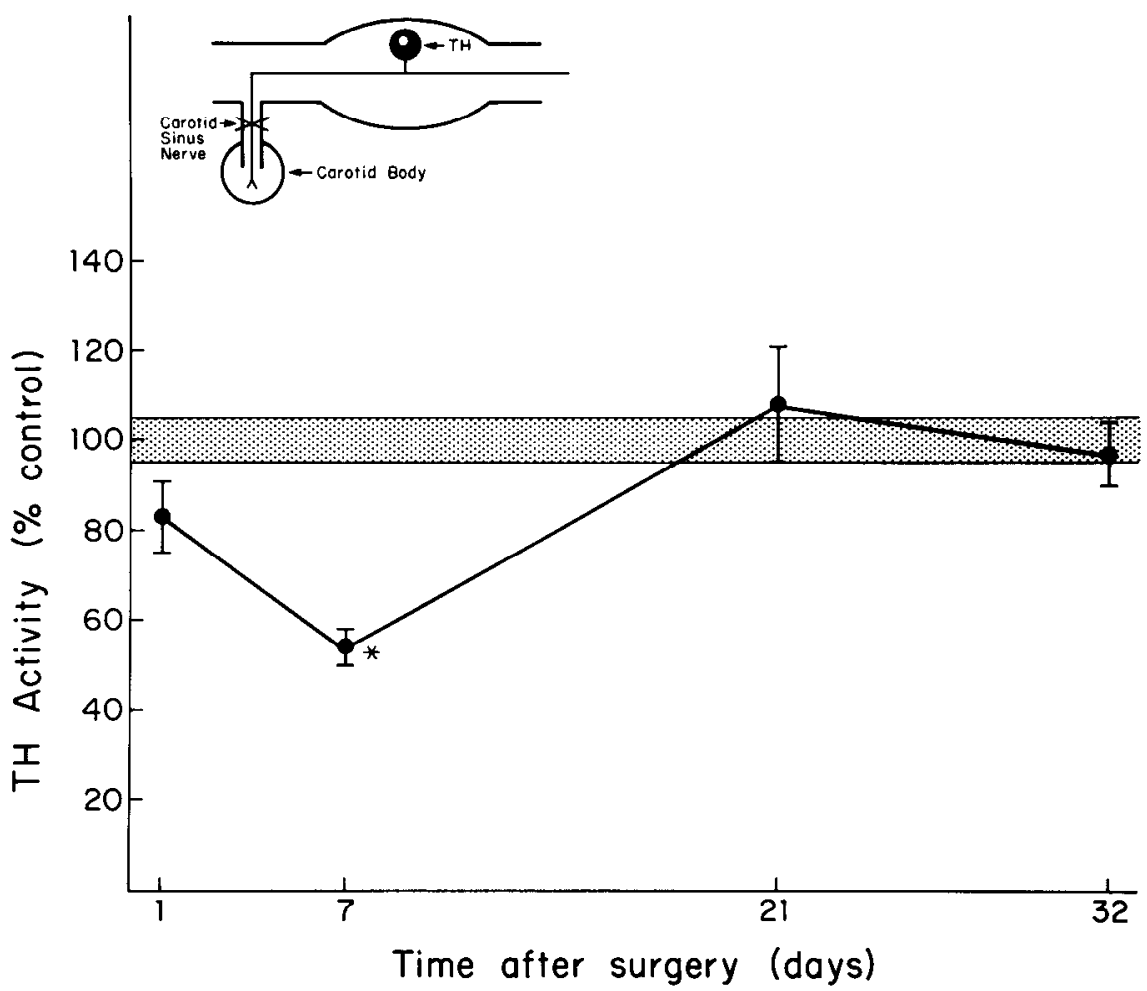

$\mathrm{TH}$, or permanent, potentially indicating cell death. To distinguish between these possibilities, TH activity was measured for up to 1 month after CSN transection (Fig. 3). In confirmation of previous findings, enzyme activity was reduced by approximately $50 \% 1$ week after surgery. This represented a reduction in specific TH activity, since total ganglion soluble protein was not significantly different from controls $[12.0 \pm 1.6(\mathrm{SEM}) \mu \mathrm{g}$ protein per operated ganglion vs $10.0 \pm 0.6 \mu \mathrm{g}$ per control; $p>$ 0.05 ). As previously reported (Katz et al., 1983), this drop in catalytic activity was accompanied by a disappearance of $\mathrm{TH}$ immunocytochemical staining within PG cell bodies. However, enzyme activity returned to control levels within 3 weeks after operation (Fig. 3). Similarly, TH cytochemical staining was restorcd (Fig. 4).

\section{Colchicine blockade of axonal transport}

Nerve transection may alter perikaryal metabolism through a variety of mechanisms, including interruption of nerve impulse conduction and axonal transport; these two events may lead to altered perikaryal metabolism through different intracellular mechanisms. To distinguish between these possibilities, enzyme activity was measured within the PG following colchicine blockade of axonal transport in the intact CSN. Colchicine is an alkaloid that reportedly disrupts axonal transport without interfering with nerve impulse conduction (Albuquerque et al., 1972). In these experiments, a colchicine-impregnated Silastic rubber cuff was placed around the nerve for 1 week. In control animals, a "blank" cuff, containing no drug, was used. As shown in Figure 5, colchicine application resulted in an approximate $40 \%$ reduction in petrosal TH activity after 1 week, compared to controls.

\section{Glossopharyngeal nerve rhizotomy}

Recent studies indicate that under certain conditions, such as chronic NGF deprivation, central, as well as peripheral sensory projections may play a role in regulation of adult perikaryal metabolism (Johnson and Yip, 1985). To define the role of central ganglion cell processes in sensory CA regulation, petrosal 


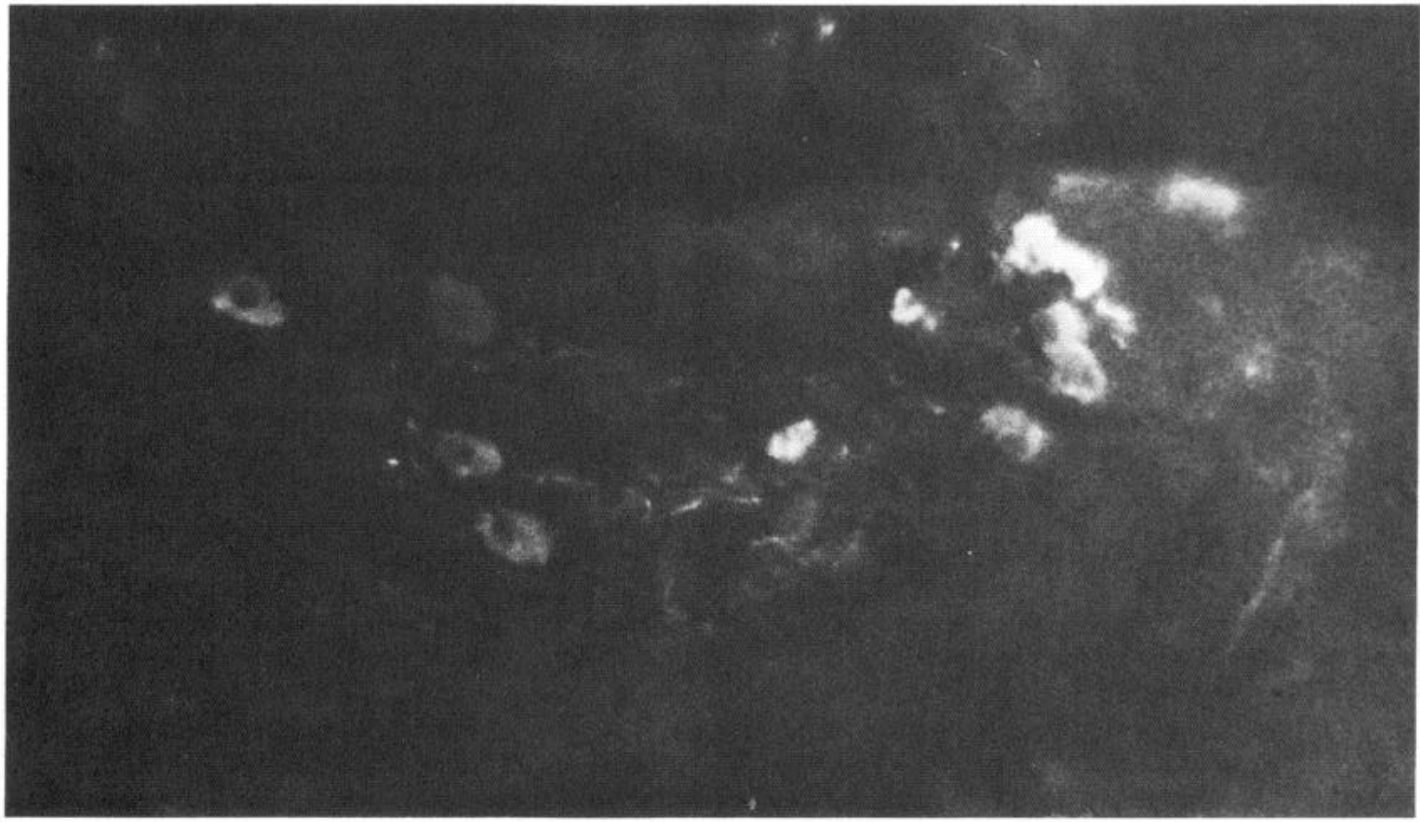

Figure 4. TH-immunoreactive PG neurons, one month after transection of the ipsilateral CSN.

TH was assayed 1 week after extracranial glossopharyngeal root transection. Ganglion cells were lesioned central to their perikarya, leaving connections to the periphery intact. TH catalytic activity in rhizotomized ganglia was not significantly different from controls 1 week after surgery (Fig. 6). Moreover, immunocytochemical staining of rhizotomized ganglia revealed abundant TH-immunoreactive sensory cell bodies, in marked contrast to the disappearance of staining seen after peripheral nerve transection (data not shown).

\section{Discussion}

The recognition that functionally and anatomically diverse neurons use the same transmitters raises central issues concerning commonalities and differences in phenotypic organization. Do similar mechanisms govern transmitter expression and metabolism in very different populations? The recent discovery that functional CA traits are expressed by primary sensory neurons (Katz et al., 1983; Price and Mudge, 1983), as well as the traditional sympathoadrenal and central cells, allows comparison

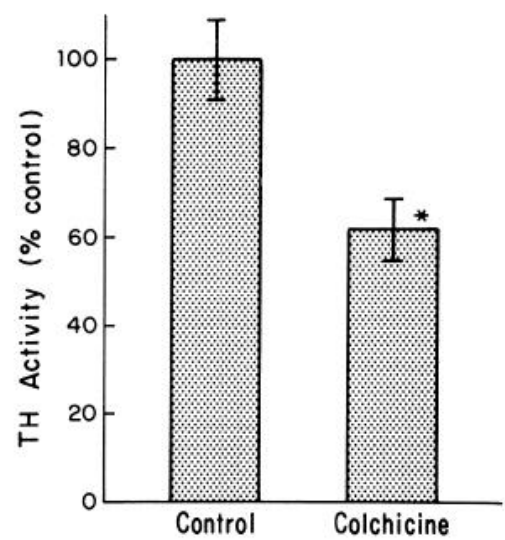

Figure 5. Effect of colchicine application to the CSN on petrosal TH catalytic activity. Enzyme activity is expressed as a percentage of control, using ganglia from age-matched animals receiving a "blank" nerve cuff for comparison. Each bar represents the mean \pm SEM for six to eight determinations. ${ }^{*} p<0.01$. of regulatory mechanisms among these groups. The apparent structural identity of individual CA molecular traits among these different neurons suggests that regulatory mechanisms may also be shared. Sensory and sympathetic TH, for example, exhibit immunoreactivity to the same highly specific antiserum, and display similar catalytic activities under identical assay conditions (Katz et al., 1983).

Multiple extracellular stimuli and intracellular mechanisms regulate individual CA traits, even within the same cell (Ciaranello et al., 1976). The present study was designed to begin elucidating sensory $\mathrm{CA}$ regulation and compare regulatory mechanisms among sensory, sympathoadrenal, and central CA cells. In view of the well-defined role of target tissues in sympathetic CA regulation, we initially examined sensory target innervation and target regulation.

\section{Retrograde labeling studies}

Retrograde fluorescent tracing, combined with TH immunocytochemistry, was used to identify peripheral targets innervated

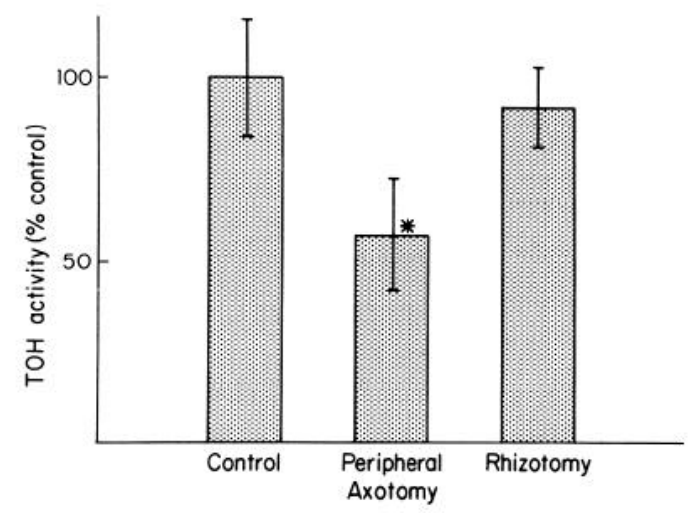

Figure 6. Comparison of rhizotomy and peripheral axotomy effects on petrosal TH activity 1 week after surgery. Enzyme activity is expressed as a percentage of control, using ganglia from age-matched animals (3-4 weeks old) for comparison. Each point represents the mean \pm SEM for $16-20$ determinations. ${ }^{*} p<0.05$. 
by CA sensory neurons. Our observations indicated that over $90 \%$ of TH-containing petrosal sensory neurons project peripherally in the CSN. Most of these cells appear to terminate in the carotid body, as indicated by the large proportion (84\%) of double-labeled, TH-positive petrosal neurons following bisbenzamide infusion into this structure. These data are consistent with the electron-microscopic localization of large dense-cored vesicles, typical of CA axon terminals, in CSN afferent fibers within the rat carotid body (McDonald, 1975). Moreover, CSN afferents make synaptic contact with Type 1 carotid body glomus cells (McDonald, 1975), suggesting that CA petrosal neurons transmit chemoreceptor information to the CNS. A small number of CA petrosal neurons may innervate the carotid sinus, also a target of the CSN, since carotid body infusion did not label as many TH-positive cells as application to the CSN itself ( 84 vs $93 \%$, respectively).

In marked contrast, less than $3 \%$ of petrosal CA neurons projected in other glossopharyngeal nerve branches. This very low incidence of bisbenzamide/TH double-labeling cannot be ascribed to methodologic artifacts, such as failure of dye uptake or transport in the glossopharyngeal nerve, since the total number of retrogradely labeled ganglion cells in these experiments exceeded that seen after CSN or carotid body labeling (Table 1).

In view of the diversity of target tissues innervated by the PG as a whole, these findings demonstrate a striking correlation between CA expression and innervation of a specific cardiovascular sensory target, the carotid body. Similarly, serotonergic sensory neurons in the cat nodose ganglion project selectively to the aortic arch (Gaudin-Chazal et al., 1983). Consequently, innervation of cardiovascular targets appears to be associated with monoaminergic phenotypic expression in sensory neurons. In contrast, expression of other transmitters, such as neuropeptides (Katz and Karten, 1980), has not generally been correlated with specific patterns of sensory target innervation. Substance P-containing sensory fibers, for example, are widely distributed in cutaneous (Hökfelt et al., 1975), gastrointestinal (Brodin, 1981), cardiopulmonary (Helke et al., 1980; Nilsson et al., 1977), and other peripheral targets (sce, however, Hunt and Rossi, 1985). Therefore, different mechanisms may govern expression of monoaminergic and peptidergic traits in sensory neurons.

\section{Effects of axotomy on sensory $\mathrm{TH}$}

Transection of peripheral ganglion cell processes markedly decreased TH catalytic activity (Fig. 4) and immunoreactivity (data not shown) after 1 week, as previously described (Katz et al., 1983). Total protein was unchanged, indicating that TH specific activity was reduced. Activity recovered (to levels observed in age-matched, sham-operated control ganglia) within 3 weeks. Recovery of $\mathrm{TH}$ immunocytochemical positivity paralleled the increase in catalytic activity, supporting the contention that the CA neurons remain viable after axotomy, re-expressing normal enzyme levels. Axotomy may, however, cause limited cell death in some adult sensory neuron populations (Aldskogius and Arvidsson, 1978). Therefore, we cannot rule out the possibility that enhanced production of TH by reduced numbers of surviving neurons accounted for the recovery of ganglion enzyme levels.

Decreased activity of CA biosynthetic enzymes has also been reported in sympathetic (Cheah and Geffen, 1973; Kessler and Black, 1979) and central (Ross et al., 1975) neurons after axotomy. The similarity of responses among sensory, sympathetic, and central neurons is particularly striking in view of the wholly dissimilar functional cellular geometries among these groups. In petrosal sensory neurons, peripheral lesions interrupt the distal axon between the source of afferent input to the cell (i.e., the carotid body) and the perikaryon. In central and sympathetic neurons, however, axotomy interrupts communication between perikaryon and efferent target. Consequently, peripheral sensory axons, postganglionic sympathetic fibers, and CNS axons may play similar roles in regulating perikaryal CA metabolism, independent of the direction of impulse traffic.

The marked decrease in petrosal $\mathrm{TH}$ after peripheral axotomy raises the possibility that target tissues influence sensory $\mathrm{CA}$ traits, as in sympathetics. For example, sympathetic $\mathrm{TH}$ fails to develop normally in the absence of target tissues (Black, 1978). Further, TH in mature sympathetics is decreased after axotomy or colchicine blockade of axonal transport (Kessler and Black, 1979). Considerable evidence indicates that NGF, synthesized in sympathetic targets and retrogradely transported to sympathetic perikarya (Thoenen and Barde, 1980), mediates target effects on sympathetic CA traits. Similar mechanisms, involving transport of factors elaborated by targets, may regulate sensory CA traits as well.

To approach this problem, we examined the effect of colchicine blockade of axonal transport in the CSN on petrosal TH. Colchicine application mimicked the effect of axotomy, reducing ganglion TH activity by $50 \%$ after 1 week. Consequently, axonal transport mechanisms may normally mediate regulatory interactions between CA sensory neurons and the periphery. However, since colchicine reportedly may damage some sensory fibers (Csillik and Knyihar-Csillik, 1982), we cannot exclude the possibility that nerve degeneration, rather than specific disruption of axonal transport, accounted for our observations. Similarly, colchicine may have produced toxic sequelae unrelated to blockade of axonal transport. However, immunohistochemical analysis failed to reveal cytopathologic changes in CA glomus cells adjacent to the PG, although these cells were probably exposed to colchicine diffusing from the application site. In contrast, TH immunoreactivity was selectively depleted in petrosal sensory neurons, projecting in the colchicine-treated nerve.

Our observations suggest that axonal transport mediates regulatory interactions between CA sensory neurons and the periphery, paralleling the regulation of sympathetic CA characters. Although we cannot yet distinguish between a role for orthoand retrograde transport in sensory CA regulation, our data suggest that common regulatory mechanisms may be shared by $\mathrm{CA}$ sensory and sympathetic neurons. At the same time, however, subtle differences may also exist. For example, unlike sensory CA neurons, sympathetic TH fails to recover fully after a peripheral lesion (Cheah and Geffen, 1973; Kessler and Black, 1979); this apparently irreversible loss of activity has becn attributed to actual disappearance of ganglion cells (Cheah and Geffen, 1973). This difference may be related to differences between sympathetic and sensory neuron morphology. For example, sensory neurons may receive trophic support from central projections that fosters recovery after peripheral axotomy. This possibility is consistent with recent studies demonstrating that central connections enhance sensory survival in NGF-deprived adults (Johnson and Yip, 1985). In the absence of analogous supplementary trophic support, sympathetic neurons may suffer irreversible changes after axotomy. In central CA neurons, on the other hand, biosynthetic enzyme activity does recover fully after axotomy (Ross et al., 1975), suggesting similarities to sensory regulation. Common extracellular factors, available to CNS and sensory neurons, could provide a basis for these observations. In aggregate, these data suggest that extracellular regulation of CA characteristics is not governed by perikaryal location, but rather by the entire range of tissue environments with which neuronal processes interact.

To define the role of central projections in regulation of sensory CA traits, we examined the effect of glossopharyngeal rhizotomy on petrosal TH. Rhizotomy did not alter enzyme activity. Similarly, in mature peptidergic sensory neurons, transection of peripheral, but not central, processes decreases perikaryal substance P (Jessell et al., 1979). Nonetheless, central 
ganglion cell processes may maintain normal perikaryal metabolism when interactions with the periphery have been perturbed (Johnson and Yip, 1985).

More generally, our studies suggest that targets rcgulate CA metabolism in sensory, as well as sympathetic and central neurons. In both sensory and sympathetic neurons, where experimental manipulation has been possible, the peripheral axon, and axonal transport, in particular, apparently regulate perikaryal CA metabolism. It may be tentatively concluded that at least some extra- and intraneuronal regulatory mechanisms are similar in diverse CA neurons. In turn, these interpretations suggest that common features do, indeed, exist in the organization of CA phenotypic characteristics in diverse populations. We are presently examining underlying molecular mechanisms to define specific loci governing expression of common features.

\section{References}

Albuquerquc, E. S., J. E. Warnick, J. R. Tasse, and F. M. Sansone (1972) Effects of vinblastine and colchicine on neural regulation of the fast and slow skeletal muscles of the rat. Exp. Neurol. 37: 607634.

Aldskogius, H., and J. Arvidsson (1978) Nerve cell degeneration and death in the trigeminal ganglion of the adult rat following peripheral nerve transection. J. Neurocytol. 7: 229-250.

Black, I. B. (1978) Regulation of autonomic development. Annu. Rev. Neurosci. 1: 183-214.

Brodin, E., J. Alumets, R. Hakanson, S. Leander, and F. Sundler (1981) Immunoreactive substance $\mathbf{P}$ in chicken gut: Distribution, development and possible functional significance. Cell Tissue Res. 216:455469.

Cheah, T. B., and L. B. Geffen (1973) Effects of axonal injury on norepinephrine, tyrosine hydroxylase and monoamine oxidase levels in sympathetic ganglia. J. Neurobiol. 4: 443-452.

Ciaranello, R. D., F. G. Wooten, and J. Axelrod (1976) Regulation of dopamine-beta-hydroxylase in rat adrenal glands. J. Biol. Chem. 250: 3204-3211.

Csillik, B., and E. Knyihar-Csillik (1982) Reversibility of microtubule inhibitor-induced transganglionic degenerative atrophy of central terminals of primary nociceptive neurons. Neuroscience 7: 1149-1154.

Gaudin-Chazal, G. P. Portalier, J. J. Puizillout, and D. Vigier (1983) Simultaneous visualization of aortic and $\left[{ }^{3} \mathrm{H}\right] 5$-hydroxytryptamineaccumulating cell bodies in the nodose ganglion of the cat. J. Physiol. (Iond.) 337: 321-330.

Haycock, J. W., J. A. Meligeni, W. F. Bennett, and J. C. Waymire (1982) Phosphorylation and activation of tyrosine hydroxylase mediate the acetylcholine-induced increase in catecholamine biosynthesis in adrenal chromaffin cells. J. Biol. Chem. 257: 12641-12648.

Helke, C. J., T. M. O'Donohue, and D. M. Jacobowitz (1980) Substance $P$ as a baro- and chemoreceptor afferent neurotransmitter: Immunocytochemical and neurochemical evidence in the rat. Peptides $1: 1-9$.
Hökfelt, T., J.-O. Kellerth, G. Nilsson, and B. Pernow (1975) Experimental immunohistochemical studies on the localization and distribution of substance $P$ in cat primary sensory neurons. Brain Res. 100: 235-252

Hunt, S. P., and J. Rossi (1985) Peptide- and non-peptide-containing unmyelinated primary afferents: The parallel processing of nociceptive information. Phil. Trans. R. Soc. Lond. [Biol.] 308: 283-289.

Jessell, T., A. Tsunoo, I. Kanazawa, and M. Otsuka (1979) Substance P: Depletion in the dorsal horn of rat spinal cord after section of the peripheral processes of primary sensory neurons. Brain Res. 168:247259.

Johnson, E. M., and H. K. Yip (1985) Central nervous system and peripheral nerve growth factor provide trophic support critical to mature sensory neuronal survival. Nature 314: 751-752.

Jonakait, G. M., K. A. Markey, M. Goldstein, and I. B. Black (1984) Transient expression of selected catecholaminergic traits in cranial sensory and dorsal root ganglia of the embryonic rat. Dev. Biol. 101: 51-60.

Katz, D. M., and H. J. Karten (1980) Substance P in the vagal sensory ganglia: Localization in cell bodies and pericellular arborizations. J. Comp. Neurol. 193: 549-564.

Katz, D. M., K. A. Markey, M. Goldstein, and I. B. Black (1983) Expression of catecholaminergic characteristics by primary sensory neurons in the normal adult rat in vivo. Proc. Natl. Acad. Sci. USA 80: 3526-3530.

Kessler, J. A., and I. B. Black (1979) The role of axonal transport in the regulation of enzyme activity in sympathetic ganglia of adult rats. Brain Res. 171: 415-424.

LeDouarin, N. M., J. Smith, and C. S. LeLièvre (1981) From the neural crest to the ganglia of the peripheral nervous system. Ann. Rev. Physiol. 43: 653-671.

Mallet, J., N. Faucon-Biguet, M. Buda, A. Lamouroux, and D. Samolyk (1983) Detection and regulation of the tyrosine hydroxylase mRNA levels in rat adrenal medulla and brain tissues. Cold Spring Harbor Symp. Quant. Biol. 1: 305-308.

Markey, K. A., S. Kondo, L. Shenkman, and M. Goldstein (1980) Purification and characterization of tyrosine hydroxylase from a clonal pheochromocytoma cell line. Mol. Pharm. 17: 79-85.

McDonald, D. M. and R. A. Mitchell (1975) The innervation of glomus cells, ganglion cells and blood vessels in the rat carotid body: A quantitative ultrastructural analysis. J. Neurocytol. 4: 177-230.

Nilsson, G., K. Dahlberg, E. Brodin, F. Sundler, and K. Strandberg (1977) In Substance P, U. S. von Euler and B. Pernow, eds., pp. 5781, Raven, New York.

Price, J., and A. W. Mudge (1983) A subpopulation of rat dorsal root ganglion neurones is catecholaminergic. Nature 301: 241-243.

Ross, R. A., T. H. Joh, and D. J. Reis (1975) Reversible changes in the accumulation and activities of tyrosine hydroxylase and dopamine- $\beta$-hydroxylase in neurons of nucleus locus coeruleus during the retrograde reaction. Brain Res. 92: 57-72.

Thoenen, H., and Y.-A. Barde (1980) Physiology of nerve growth factor. Physiol. Rev. 60: 1284-1385. 